\title{
The natural emergence of asymmetric tree-shaped pathways for cooling of a non-uniformly heated domain
}

\author{
Erdal Cetkin ${ }^{1}$ and Alessandro Oliani ${ }^{1,2}$ \\ ${ }^{1}$ Department of Mechanical Engineering, Izmir Institute of Technology, Urla, Izmir 35430, Turkey \\ ${ }^{2}$ Department of Engineering “Enzo Ferrari," Università di Modena e Reggio Emilia, Modena, Italy
}

(Received 29 May 2015; accepted 1 July 2015; published online 9 July 2015)

\begin{abstract}
Here, we show that the peak temperature on a non-uniformly heated domain can be decreased by embedding a high-conductivity insert in it. The trunk of the high-conductivity insert is in contact with a heat sink. The heat is generated non-uniformly throughout the domain or concentrated in a square spot of length scale $0.1 L_{0}$, where $L_{0}$ is the length scale of the non-uniformly heated domain. Peak and average temperatures are affected by the volume fraction of the high-conductivity material and by the shape of the high-conductivity pathways. This paper uncovers how varying the shape of the symmetric and asymmetric high-conductivity trees affects the overall thermal conductance of the heat generating domain. The tree-shaped high-conductivity inserts tend to grow toward where the heat generation is concentrated in order to minimize the peak temperature, i.e., in order to minimize the resistances to the heat flow. This behaviour of high-conductivity trees is alike with the root growth of the plants and trees. They also tend to grow towards sunlight, and their roots tend to grow towards water and nutrients. This paper uncovers the similarity between biological trees and high-conductivity trees, which is that trees should grow asymmetrically when the boundary conditions are non-uniform. We show here even though all the trees have the same objectives (minimum flow resistance), their shape should not be the same because of the variation in boundary conditions. To sum up, this paper shows that there is a high-conductivity tree design corresponding to minimum peak temperature with fixed constraints and conditions. This result is in accord with the constructal law which states that there should be an optimal design for a given set of conditions and constraints, and this design should be morphed in order to ensure minimum flow resistances as conditions and constraints change. (C) 2015 AIP Publishing LLC.
\end{abstract}

[http://dx.doi.org/10.1063/1.4926620]

\section{INTRODUCTION}

Technology evolves toward to smaller and more powerful devices, especially electronic equipment. In addition to limitation in size, the volumetric heating rate of these equipment increases due to the trend of miniaturization. ${ }^{1-4}$ Overall, with miniaturization comes an expense: smaller parts are more difficult to cool with the traditional cooling methods and coolants. Therefore, cooling methods have transformed from natural convection to forced convection, and coolants have transformed from one phase coolants to two phase and nanofluid coolants in the literature. ${ }^{5-10}$ Literature also shows that embedding high-conductivity pathways increases the overall thermal conductance of a heat generating domain. However, current literature only discusses how this high-conductivity material should be distributed in a uniformly heated domain. ${ }^{1-15}$ Here, we uncover how the high-conductivity material should be placed when the heat generation is non-uniform. Here, we use constructal theory in order to uncover how the shape of the highconductivity inserts should be in order to minimize maximum temperature in a non-uniformly heated domain.

Constructal law stated in 1996 by Adrian Bejan is as follows: "For a finite-size system to persist in time (to live), it must evolve in such a way that it provides easier access to the imposed currents that flow through it."1,16,17 This law illustrated that the effect of design is applicable on both animate (biology, zoology, animal locomotion) $)^{1,16-18}$ and inanimate (physics, geology, geophysics, engineering). 1,16,17,19-24 This law is a tool for one to calculate what the shape of a system should be without observation, i.e., pure theory. Therefore, the constructal law is used in the current discussion. In this paper, we uncover how the shape of the embedded high-conductivity material should be morphed in order to minimize the maximum temperature and provide uniform temperature distribution on a non-uniformly heated domain, i.e., heat generation is a function of spatial coordinate. In addition, the effect of concentrated heat generation on the peak temperature of the non-uniformly heated domain is also documented.

\section{NUMERICAL METHOD}

Consider a two-dimensional conductive domain with length of $L_{0}$ and height of $H_{0}$, Fig. 1 . The thermal conductivity of the domain of size $L_{0} \times H_{0}$ is $k_{l}$. A rectangular domain of size $B_{0} \times D_{0}$ with the thermal conductivity of $k_{h}$ is embedded in the domain of thermal conductivity $k_{l}$. Heat is generated only in the low-conductivity domain with a generation rate of $\mathrm{q}^{\prime \prime \prime}$. The volume of the low-conductivity domain is fixed, so is the high-conductivity domain volume. The volume fraction of the high-conductivity domain over the heat generating domain is $\phi=B_{0} D_{0} / L_{0} H_{0}$. 


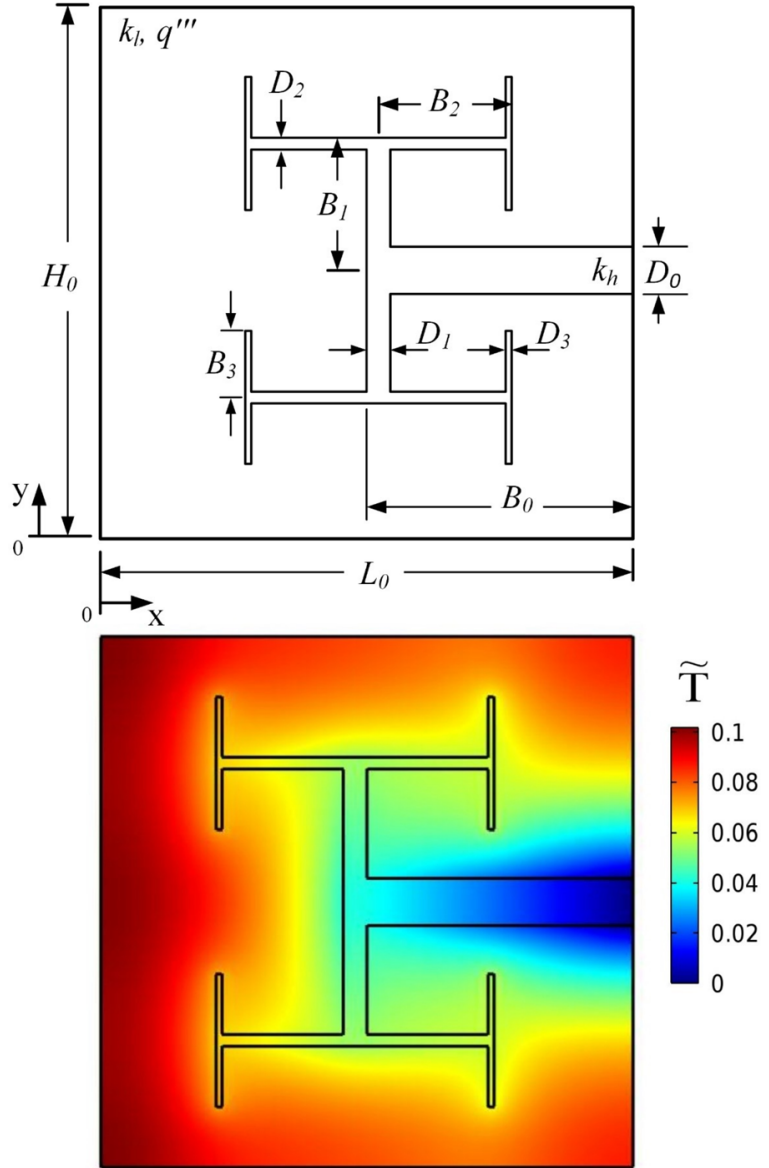

FIG. 1. Heat generating domain with embedded high-conductivity pathways: (a) geometry and (b) temperature distribution.

The trunk of the high-conductivity domain (diameter of $D_{0}$ ) is fixed at a prescribed temperature at $\mathrm{x}=L_{0}$, i.e., heat sink, Fig. 1. Other outer boundaries are symmetry boundaries, i.e., the domain of size $L_{0} \times H_{0}$ is an elemental area of a greater domain. Heat transfer in the conductive domain is governed by the energy equation. For isotropic material with constant properties and steady state, two-dimensional energy equation for the heat generating domain can be written as

$$
\frac{\partial^{2} T}{\partial x^{2}}+\frac{\partial^{2} T}{\partial y^{2}}+\frac{q^{\prime \prime \prime}}{k_{l}}=0
$$

There is no heat generation in the high-conductivity domain; therefore, the energy equation for the high-conductivity domain becomes

$$
\frac{\partial^{2} T}{\partial x^{2}}+\frac{\partial^{2} T}{\partial y^{2}}=0
$$

The generated heat inside the low-conductivity domain is transferred from it to high-conductivity pathways. Continuity of heat is required at the intersection of low- and high-conductivity domains, which is

$$
\left.k_{l}\left(\frac{\partial T}{\partial n}\right)\right|_{l}=\left.k_{h}\left(\frac{\partial T}{\partial n}\right)\right|_{h},
$$

where $\mathrm{T}$ and $\mathrm{n}$ are the temperature and normal direction.
The dimensionless length scale and the dimensionless temperature are

$$
(\widetilde{x}, \widetilde{y})=(x, y) / A_{0}^{1 / 2} \quad \widetilde{T}=\frac{T-\left.T\right|_{\left(L_{0}, 0\right)}}{q^{\prime \prime \prime} A_{0} / k_{l}},
$$

where $A_{0}$ is the area of the conductive domain of size $L_{0}$ $\times H_{0}$. Dimensionless energy equations for low-conductivity and high-conductivity domains become

$$
\begin{gathered}
\frac{\partial^{2} \widetilde{T}}{\partial \widetilde{x}^{2}}+\frac{\partial^{2} \widetilde{T}}{\partial \widetilde{y}^{2}}+1=0, \\
\frac{\partial^{2} \widetilde{T}}{\partial \widetilde{x}^{2}}+\frac{\partial^{2} \widetilde{T}}{\partial \widetilde{y}^{2}}=0 .
\end{gathered}
$$

The dimensionless heat continuity between the high- and low-conductivity materials is

$$
\left.\left(\frac{\partial \widetilde{T}}{\partial \widetilde{n}}\right)\right|_{l}=\left.\widetilde{k}\left(\frac{\partial \widetilde{T}}{\partial \widetilde{n}}\right)\right|_{h}
$$

where $\widetilde{k}=k_{h} / k_{l}$.

The dimensionless energy equation is solved using a finite element software. ${ }^{25}$ Boundary layer meshes are applied to the boundaries in order to minimize the numerical error caused by variation of the temperature gradient near the walls. Table I shows that the relative error $\left(\left|\widetilde{T}_{\text {peak }}{ }^{n+1}-\widetilde{T}_{\text {peak }}{ }^{n}\right|\right) / \widetilde{T}_{\text {peak }}{ }^{n}$ becomes less than $10^{-4}$ as the number of the mesh elements is 2711. In addition, 1st law of thermodynamics states that the energy should be conserved. Therefore, in steady state, the heat which is generated on the domain should be equal to the heat which is transferred from the heat sink surface. The error between the imposed heat transfer rate and the calculated value from the numerical simulation is $0.3 \% \quad\left(\left\{\left|\mathrm{q}_{\mathrm{sim}}-\mathrm{q}\right|\right\} /\right.$ $q=0.003048)$ with 2711 mesh elements. Therefore, it is concluded that the results are mesh independent and valid in accordance with the 1st law of thermodynamics.

In addition, the validation of the numerical method is also checked by comparing the current results with the results of Ref. 11. The same conditions and assumptions of Ref. 11 are used, such as $\phi=0.1, H_{0} / L_{0}=1, D_{0} / B_{0}=0.1$. Table II shows how the peak temperature is affected by the dimensionless thermal conductivity ratio in the current study and in Ref. 11. The error in between the current study and Ref. 11 is always less than $0.4 \%$. Therefore, it is concluded that the current numerical model is validated with the consideration of the 1st law of thermodynamics and results of Ref. 11.

TABLE I. Mesh dependency test.

\begin{tabular}{lcccc}
\hline \hline $\begin{array}{l}\text { Mesh } \\
\text { number }\end{array}$ & $\widetilde{T}_{\text {peak }}$ & $\left(\left|\widetilde{T}_{\text {peak }}{ }^{n+1}-\widetilde{T}_{\text {peak }}{ }^{n}\right|\right) / \widetilde{T}_{\text {peak }}{ }^{n}$ & $\mathrm{q}_{\text {sim }}$ & $\left(\left|\mathrm{q}_{\text {sim }}-\mathrm{q}\right|\right) / \mathrm{q}$ \\
\hline 465 & 0.157449 & $3.6837 \times 10^{-4}$ & 0.995555 & 0.004445 \\
1047 & 0.157516 & $2.0315 \times 10^{-4}$ & 0.996644 & 0.003356 \\
1642 & 0.157548 & $8.8862 \times 10^{-5}$ & 0.996598 & 0.003402 \\
2711 & 0.157562 & & 0.996952 & 0.003048 \\
\hline \hline
\end{tabular}


TABLE II. Validation test between current study and Ref. 11.

\begin{tabular}{lcc}
\hline \hline$\widetilde{k}$ & $\mathrm{FE}^{\mathrm{a}}$ & $\mathrm{FE}^{\mathrm{b}}$ \\
\hline 1000 & 0.128236 & 0.128579 \\
300 & 0.135924 & 0.136263 \\
100 & 0.157219 & 0.157548 \\
30 & 0.224812 & 0.225157 \\
10 & 0.374893 & 0.375647 \\
\hline \hline
\end{tabular}

${ }^{\mathrm{a}}$ Ref. 11.

${ }^{\mathrm{b}}$ Current study.

Furthermore, Figure 1(b) shows the temperature distribution with an embedded rectangular high-conductivity insert that is $10 \%$ of the domain $L_{0} \times H_{0}$. The blue and red represents minimum and maximum temperatures, respectively. This figure shows the dimensionless temperature distribution with q" " $=1$ when heat generation is uniform in the domain. The temperature is the maximum at the two corners farthest from the heat $\operatorname{sink}\left(0, \pm \mathrm{H}_{0} / 2\right)$.

\section{NON-UNIFORM HEAT GENERATION}

Consider a heat generating conductive domain on which high-conductivity material is placed in order to maximize the thermal conductance of the domain, as shown in Fig. 1. The high-conductivity thermal pathway is rectangular, with the height over length ratio of $0.1\left(D_{0} / B_{0}=0.1\right)$. Height and length of the heat-generating domain are the same, i.e., the domain is square. The volume fraction of the highconductivity material over the heat generating one is fixed at $\phi=0.1$. In addition, the ratio of the conductivities of highand low-conductivity material is $\widetilde{k}=100$, and the dimensionless temperature of the heat sink boundary is fixed at $\widetilde{T}=0$.

Cetkin $^{12}$ showed that $\mathrm{T}$ - and $\mathrm{Y}$-shaped conductive pathways increase the overall thermal conductance of a uniformly heated domain more than distributing the same amount of high-conductivity material uniformly in the domain. Therefore, uniform distribution of the highconductivity material is not discussed in this paper. Here, we uncover how $\mathrm{T}$-shaped conductive pathways should be morphed in order to minimize the peak temperature. The relation between the length of the branches is $B_{i}=B_{i-1} / 2$ (i.e., $B_{1}=B_{0} / 2$ and so on) except for $B_{2}=B_{1}$. The relation between the thickness of the branches is $D_{i}=D_{i-1} / 2$, which is the optimal thickness ratio showed analytically in Ref. 1 and numerically in Ref. 12.

The heat generation in the domain is non-uniform, such as $q^{\prime \prime \prime}=2(1-x)$ and $q^{\prime \prime \prime}=2 x$ but the overall heat generation rate in the domain is constant. In addition, uniform and constant heat generation with $q^{\prime \prime \prime}=1$ is considered. Overall heat generation rate is fixed, so is the volume fraction, i.e., the shape of the high-conductivity tree is the only variable.

Figure 2 shows how the number of tree branches affects the peak temperature for three volume fractions: $\phi_{0}=0.05,0.1$, and 0.15 . Increasing the volume fraction of the high-conductivity material decreases the peak temperature as shown in Fig. 2. In other words, the overall thermal conductance increases as $\phi$ increases. In addition, the overall heat generation rate decreases because heat is only generated in the low conductivity domain. Even though increasing the high-conductivity volume with the same rate does not decrease the peak temperature with the same rate. For example, increasing $\phi_{0}$ from 0.05 to 0.1 decreases the peak temperature by $17.15 \%$, while increasing $\phi_{0}$ from 0.1 to 0.15 decreases the peak temperature by $13.41 \%$ (with $q^{\prime \prime \prime}=$
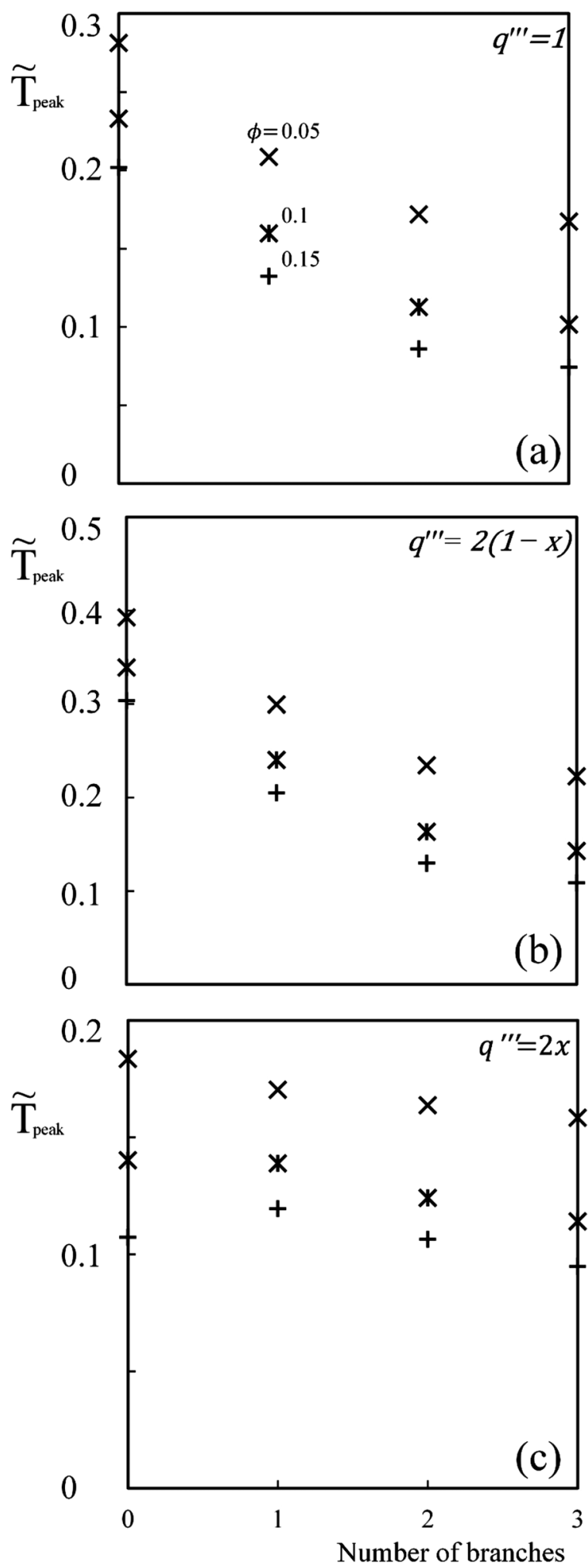

FIG. 2. $\widetilde{T}_{\text {peak }}$ relative to the number of T-shaped tree branches for uniform and non-uniform heat generation: $q^{\prime \prime \prime}=(\mathrm{a}) 1$, (b) $2(1-x)$, and (c) $2 x$ for volume fractions of $0.05,0.1$, and 0.15 . 
$2(1-x)$ and $\mathrm{N}=0)$. This shows the return of adding more high-conductivity material diminishes as the volume fraction increases.

Figure 2(a) shows how the peak temperature is affected by the variation in volume fraction and by the number of bifurcations when the heat generation is constant and uniform, i.e., $q^{\prime \prime \prime}=1$. The peak temperature decreases as $\phi$ and $\mathrm{N}$ increase. Figures 2(b) and 2(c) show how the peak temperature is affected as the number of branches increases with the non-uniform heat generation, respectively, $q^{\prime \prime \prime}=2(1-x)$ and $q^{\prime \prime \prime}=2 x$. Similar to uniform heat generation case of Figure 2(a), increasing the number of branches decreases the peak temperature with non-uniform heat generation concentrated near the trunk of the high conductivity tree. As the number of branches increases the decrease in the peak temperature diminishes, i.e., the peak temperature reduces by $29.1 \%$ as $\mathrm{N}$ increases from 0 to 1 , and it reduces by $12.57 \%$ as $\mathrm{N}$ increases from 2 to 3 when $\phi_{0}=0.1$. Unlike Figures 2(a) and 2(b), the peak temperature increases as $\mathrm{N}$ increases from 0 to 1 with $\phi_{0}=0.15$ and remains almost constant with $\phi_{0}=0.1$. In Figure 2(c), when $\phi_{0}=0.05$, the peak temperature decreases as $\mathrm{N}$ increases. However, increasing $\mathrm{N}$ from 1 to 3 does not change the order of peak temperature, as much as it does in Figures 2(a) and 2(b).

\section{CONCENTRATED HOT SPOT}

Consider next a concentrated hot spot on the lowconductivity heat generating domain. The non-uniform heat generation rate in the low-conductivity domain is $q^{\prime \prime \prime}=$ $1,2(1-x)$ or $2 x$. The length scale of a square shaped concentrated hot spot is $0.1 L_{0}$, and its volumetric heating rate is varied from $q^{\prime \prime \prime}=10$ to 1000 . Fixed amount of highconductivity material is placed on the domain as T-shaped trees. The trunk of the high-conductivity tree is in contact with the heat sink, i.e., trunk boundary temperature is $\widetilde{T}=0$. The ratio of the thermal conductivities of high- and lowconductivity material is fixed at $\widetilde{k}=100$.

The effect of the hot spot on temperature distribution is uncovered for three distinct hotspot location: $(\mathrm{x}, \mathrm{y})=(0.2$, $0.45),(0.05,0.85)$, and $(0.8,0.65)$. Figure 3 shows how the peak temperature is affected by the location and heating rate of the concentrated heat generating region. Figures 3(a), 3 (b), and 3(c) show that $\widetilde{T}_{\text {peak }}$ decreases as number of the high-conductivity branches increases while the amount of high-conductivity material is fixed. In addition, $\widetilde{T}_{\text {peak }}$ for the concentrated hot spot locations of $(\underset{\widetilde{T}}{\mathrm{x}} \mathrm{y})=(0.2,0.45)$ and $(0.8,0.65)$ are in the same order (i.e., $\widetilde{T}_{\text {peak }}=0.403$ and 0.477 with $q^{\prime \prime \prime}=100$ and $\mathrm{N}=2$, respectively), and they are smaller in comparison with when the hot spot is located at $(\mathrm{x}, \mathrm{y})$ $=(0.05,0.85)$ (i.e., $\widetilde{T}_{\text {peak }}=0.918$ with $q^{\prime \prime \prime}=100$ and $\left.\mathrm{N}=2\right)$. The concentrated spot location of $(\mathrm{x}, \mathrm{y})=(0.2,0.45)$ and $(0.8,0.65)$ provides shorter distance for heat to flow from the hot spot to the high-conductivity pathway. However, Figures 3(b) and 3(c) show that the difference of $\widetilde{T}_{\text {peak }}$ in between the hot spot locations decreases as $q^{\prime \prime \prime}$ decreases and $\mathrm{N}$ increases. As $q^{\prime \prime \prime}$ of the hot spot decreases, the non-uniform heat generation of low-conductivity domain becomes the governing factor. Therefore, the effect of the hot spot location
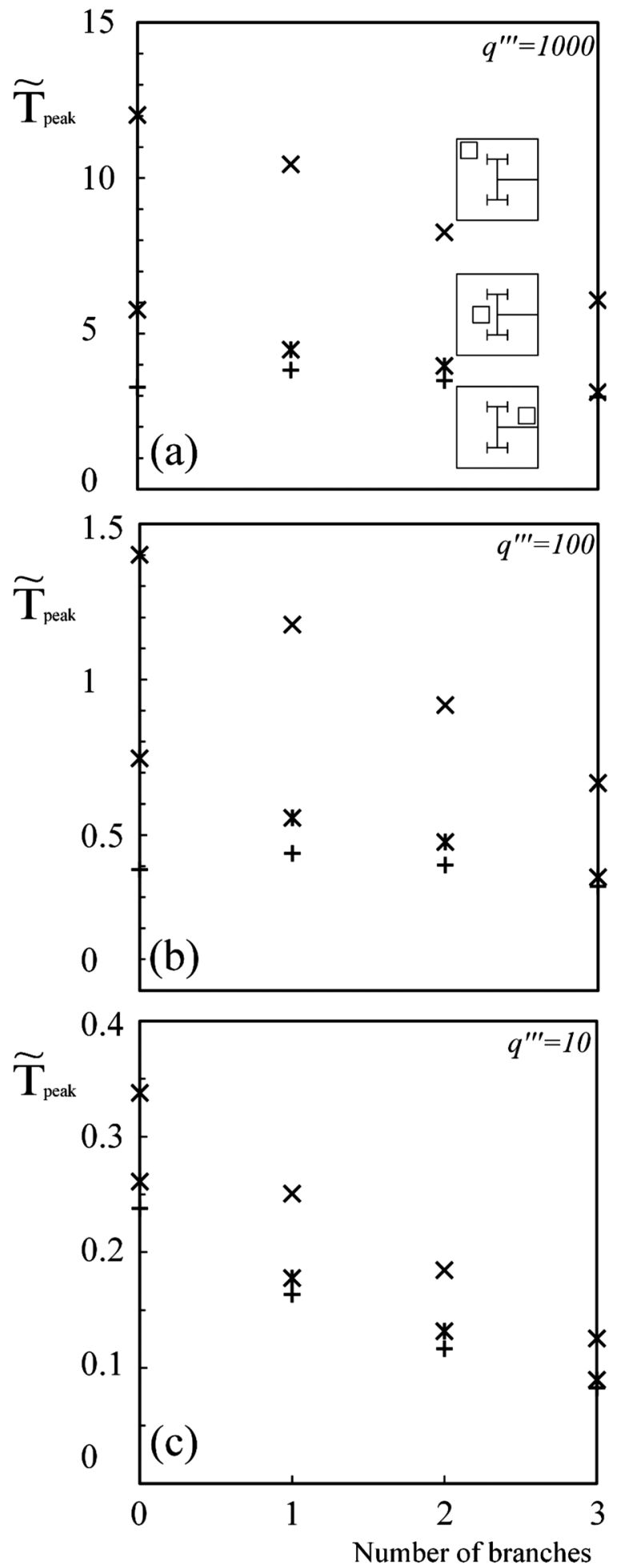

FIG. 3. $\widetilde{T}_{\text {peak }}$ relative to the number of tree branches with uniform heat generation $\left(q^{\prime \prime \prime}=1\right)$ in the low-conductivity domain and variable heat generation rate in the concentrated heat generation spot of heating rate $q^{\prime \prime \prime}=(\mathrm{a}) 1000,(\mathrm{~b}) 100$, and (c) 10 .

diminishes as $q^{\prime \prime \prime}$ of the concentrated hot spot decreases. Furthermore, the thermal conductance of the entire domain increases as $\mathrm{N}$ increases, and this decreases the effect of the hot spot location. However, Figures 3(a), 3(b), and 3(c) show that $\widetilde{T}_{\text {peak }}$ is the maximum when the hot spot is located at $(\mathrm{x}, \mathrm{y})=(0.05,0.85)$. This location is the farthest from both the heat sink and the conductive pathways. 
Figure 4 shows how the temperature distribution is affected by the location of the hot spot with uniform $\left(q^{\prime \prime \prime}=1\right)$ and non-uniform $\left(q^{\prime \prime \prime}=2(1-x)\right.$ and $\left.2 x\right)$ heat generation rates, where blue and red represent minimum and maximum temperatures, respectively. Figures 4(a) and 4(b) show how the temperature distribution varies when the hot spot location changes from $\mathrm{x}=0.2, \mathrm{y}=0.45$ to $\mathrm{x}=0.05$, $\mathrm{y}=0.85$ with $q^{\prime \prime \prime}=2(1-x)$ on the low conductivity domain when $q^{\prime \prime \prime}=10$ in the concentrated hot spot. The heat generation is concentrated in the farthest region from the heat sink, and therefore the temperature becomes the greatest when $\mathrm{x}<0.2 . \widetilde{T}_{\text {peak }}$ is smaller in Fig. 4(a) than in Fig. 4(b) because the thermal pathways are closer to the concentrated heat generating domain which increases the overall thermal conductance. Similarly, temperature distribution of Figs. 4(c) and 4(e) is more uniform and smaller in comparison with Figs. 4(d) and 4(f), respectively. In summary, Figure 4 shows that the concentrated hot spot should be placed closer to the high-conductivity pathways. However, if the locations of the hot spot are known and fixed, the high-conductivity pathways should be designed according to this constraint.

\section{ASYMMETRIC HIGH-CONDUCTIVITY TREES}

Next, consider the heat generating domain with embedded Y-shaped asymmetric high-conductivity inserts. The heat generation rate of the high-conductivity domain is $q^{\prime \prime \prime}=1$ and $q^{\prime \prime \prime}=2(1-x)$ without concentrated hot spot, and $q_{1}^{\prime \prime \prime}=1$

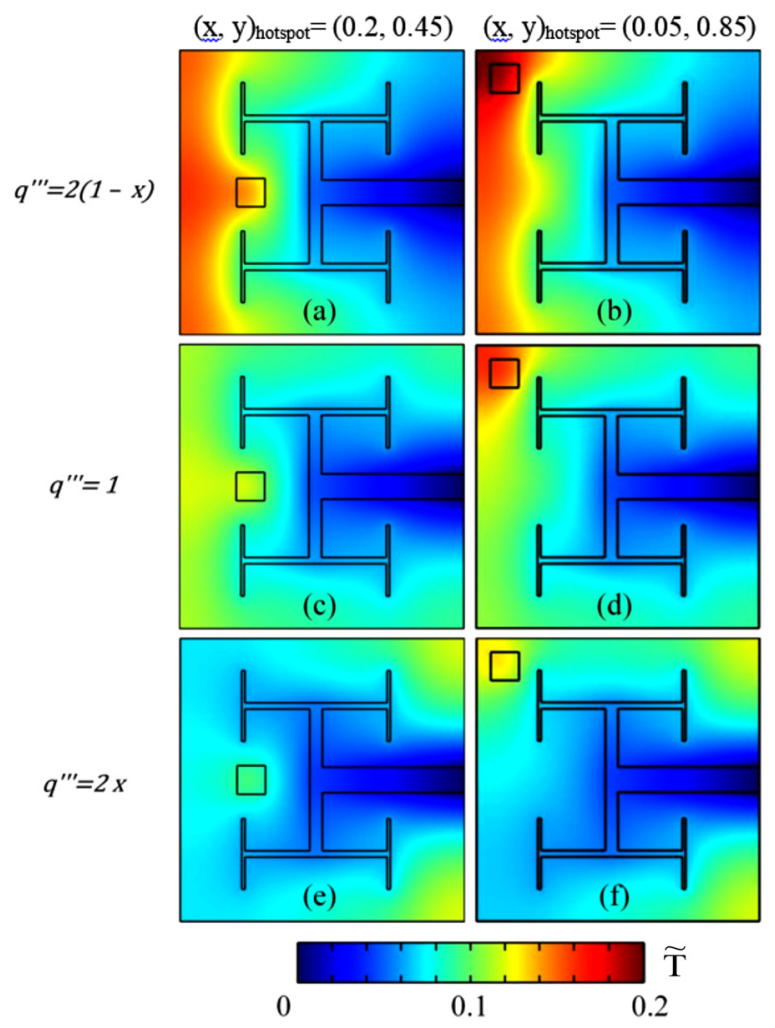

FIG. 4. Temperature distribution for hot spot locations of $(x, y)=(0.2,0.45)$ and $(0.05,0.85)$ with uniform and non-uniform heat generation rates: (a) $(\mathrm{x}, \mathrm{y})=(0.2,0.45)$ and $q^{\prime \prime \prime}=2(1-x),(\mathrm{b})(\mathrm{x}, \mathrm{y})=(0.05,0.85)$ and $q^{\prime \prime \prime}$ $=2(1-x)$, (c) $(\mathrm{x}, \mathrm{y})=(0.2,0.45)$ and $q^{\prime \prime \prime}=1$, (d) $(\mathrm{x}, \mathrm{y})=(0.05,0.85)$ and $q^{\prime \prime \prime}=1,(\mathrm{e})(\mathrm{x}, \mathrm{y})=(0.2,0.45)$ and $q^{\prime \prime \prime}=2 x$, and $(\mathrm{f})(\mathrm{x}, \mathrm{y})=(0.05,0.85)$ and $q^{\prime \prime \prime}=2 x$. with concentrated hot spot of heat generating rate of $q_{2}{ }^{\prime \prime \prime}$ $=100$, as shown in Fig. 5(a), where $q_{2}^{\prime \prime \prime}=q_{1}{ }^{\prime \prime \prime}=q^{\prime \prime \prime}$ without the concentrated heat generation. The amount of embedded high-conductivity material is fixed, and is the same in Figs. 3 and 4. First, $\alpha$ is varied from $0^{\circ}$ to $60^{\circ}$ in order to uncover how it affects $\widetilde{T}_{\text {peak }}$. Fig. 5(b) shows that increasing $\alpha$ from $0^{\circ}$ (T-shaped) to $60^{\circ}$ (Y-shaped) decreases the peak temperature. This result is in accord with the current literature. ${ }^{24}$ This decrease is not linear, as expected, because as $\alpha$ increases from $0^{\circ}$ to $40^{\circ}$ the $\mathrm{Y}$-shaped tree invades the greatest region. Then, as $\alpha$ is increased after $40^{\circ}$, the region in between the two branches gets smaller, and the thermal boundary layer of the bifurcating trees overlaps. Therefore, $\alpha$ should be in between $40^{\circ}$ and $60^{\circ}$, depending on the number of bifurcation angle and volume fraction.

Next, consider the effects of $\alpha$ and $\beta$ on $\widetilde{T}_{\text {peak }}$ simultaneously. Figure 6 shows the family of fixed $\alpha$ curves for variable $\beta$ angles with heat generation rate of $q^{\prime \prime \prime}=1$ and $q^{\prime \prime \prime}=2(1-x)$ without concentrated hot spot, and $q_{1}^{\prime \prime \prime}=1$ with concentrated hot spot of $q_{2}{ }^{\prime \prime \prime}=100$. Figure 6(a) shows how $\widetilde{T}_{\text {peak }}$ varies relative to $\alpha$ and $\beta$ when the heat generation rate is uniform $\left(q^{\prime \prime \prime}=1\right)$ without hotspot. Figure 6(a) shows that the $\widetilde{T}_{\text {peak }}$ is minimum with $\alpha=30^{\circ}$ and $\beta=40^{\circ}$. It also
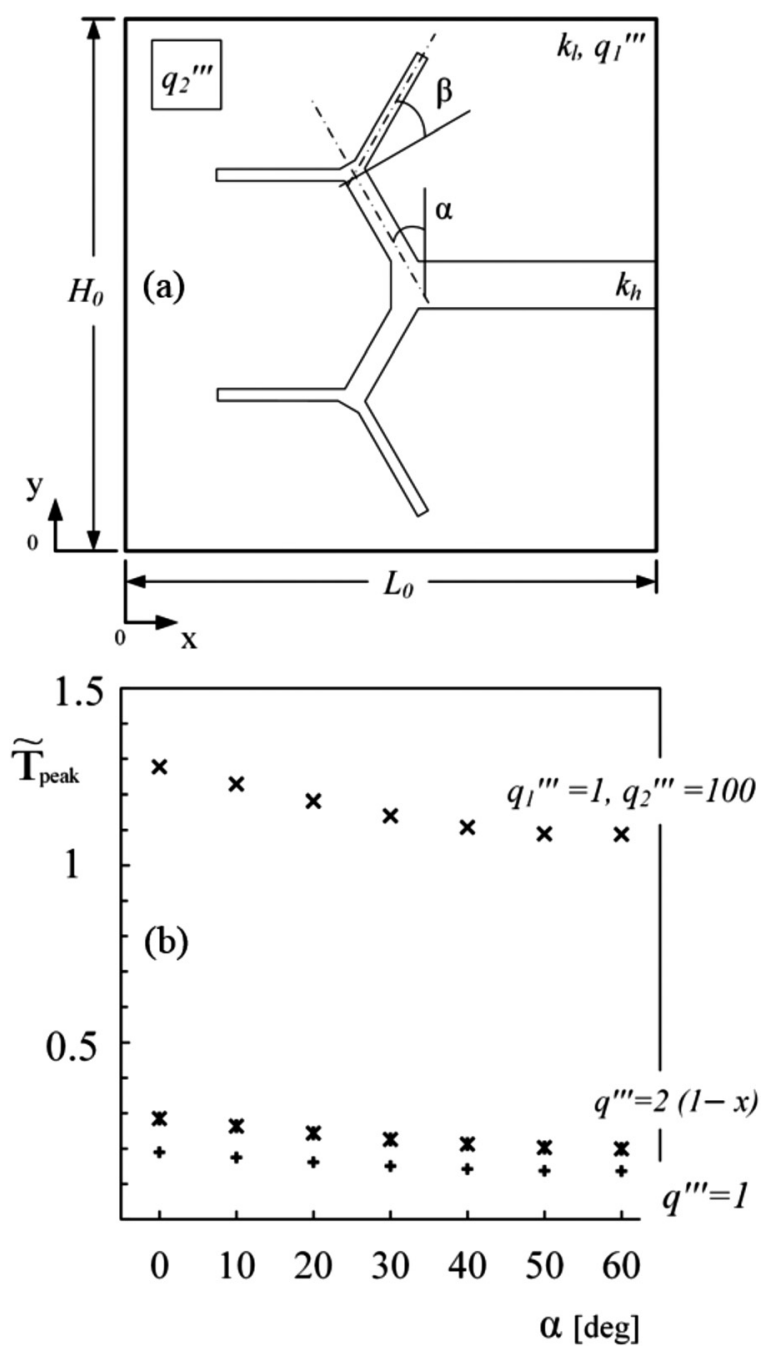

FIG. 5. Heat generating domain with embedded symmetric Y-shaped pathways: (a) geometry and (b) $\widetilde{T}_{\text {peak }}$ relative to $\alpha$. 
shows that $\widetilde{T}_{\text {peak }}$ is the smallest with $\alpha=40^{\circ}$ and $50^{\circ}$ when $\beta$ is smaller than $30^{\circ}$. Furthermore, Figure 6(a) shows that the worst performing tree-shaped design is T-shaped design (i.e., $\left.\alpha=0^{\circ}, \beta=0^{\circ}\right)$. Figure $6(\mathrm{~b})$ shows how $\widetilde{T}_{\text {peak }}$ varies relative to $\alpha$ and $\beta$ when $q^{\prime \prime \prime}=2(1-x)$ without concentrated hot spot in the domain. Figure 6(b) shows that, as both $\alpha$ and $\beta$ increase from $0^{\circ}$ to $60^{\circ}, \widetilde{T}_{\text {peak }}$ decreases. The reason of this
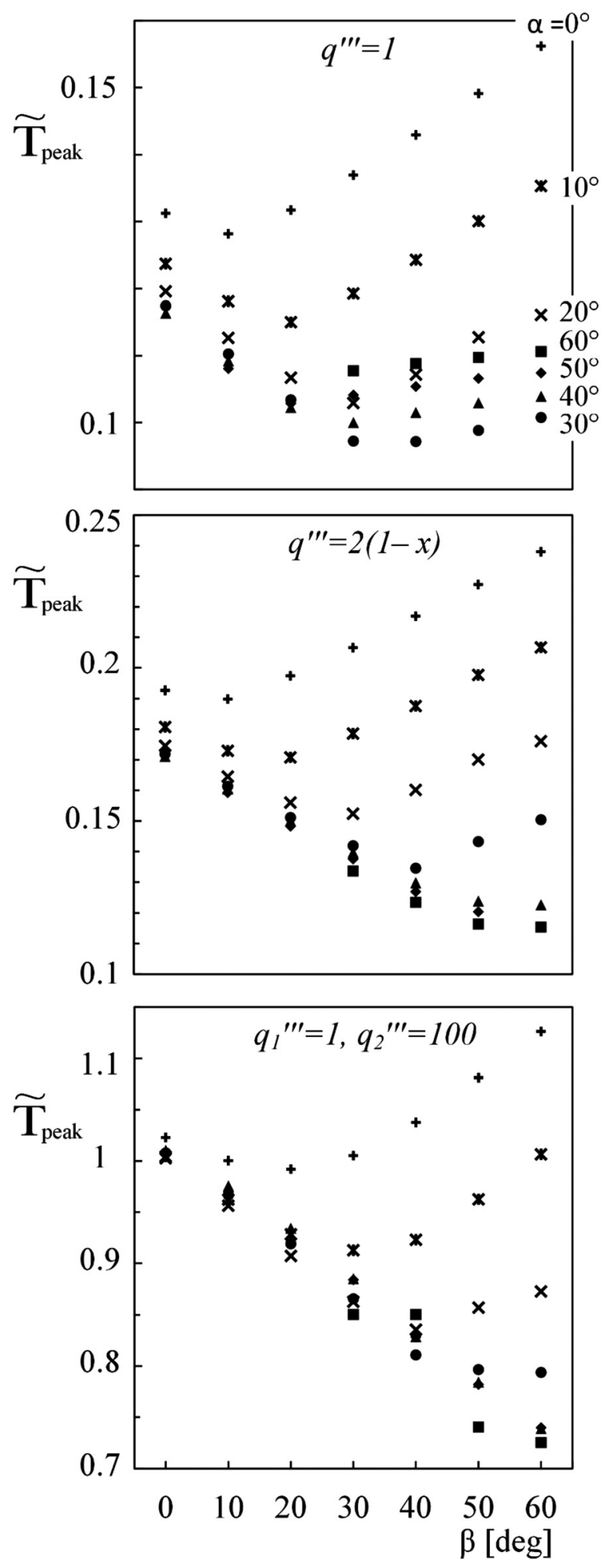

FIG. 6. $\widetilde{T}_{\text {peak }}$ relative to the Y-shaped tree branches for uniform and nonuniform heat generation: (a) $q^{\prime \prime \prime}=1$, (b) $q^{\prime \prime \prime}=2(1-x)$, and (c) $q_{1}^{\prime \prime \prime}=1$, $q_{2}^{\prime \prime \prime}=100$ with variable $\alpha$ and $\beta$. tendency is natural, because the heat generation rate is concentrated on the left side of Fig. 5(a). Therefore, Y-shaped tree of Fig. 5(a) should grow in the direction of the left side of the domain. In addition, Figure 6(c) shows how $\widetilde{T}_{\text {peak }}$ varies relative to $\alpha$ and $\beta$ with the concentrated hot spot of $q_{2}{ }^{\prime \prime \prime}=100$. The existence of the concentrated hot spot provides greater temperature on the left side of the domain, as shown in Fig. 5(a). Therefore, $\widetilde{T}_{\text {peak }}$ is minimum with $\alpha=\beta=60^{\circ}$ as shown in Fig. 6(b). However, the minimum $\widetilde{T}_{\text {peak }}$ is almost 6 times greater in Fig. 6(c) than in Fig. 6(b). Moreover, there is a $\beta$ value corresponding to the smallest $\widetilde{T}_{\text {peak }}$ for a given $\alpha$ in Fig. 6(b) and increasing $\beta$ increases $\widetilde{T}_{\text {peak }}$ after this value. However, this tendency is not smooth in Fig. 6(c) as it is in Fig. 6(b). The reason for the unpredictable nature of this tendency is due to the non-uniformity of the heat generation, which is concentrated on a small region. Therefore, if a tree branch gets closer to this region, $\widetilde{T}_{\text {peak }}$ decreases, i.e., not necessarily a tree grows in the direction of left side of the domain, but a tree grows forward to concentrated hot spot or at least one branch of it grows toward where the hot spot is located.

Last, consider the conductive domain of Figure 7(a) with embedded high-conductive insert and with non-uniform
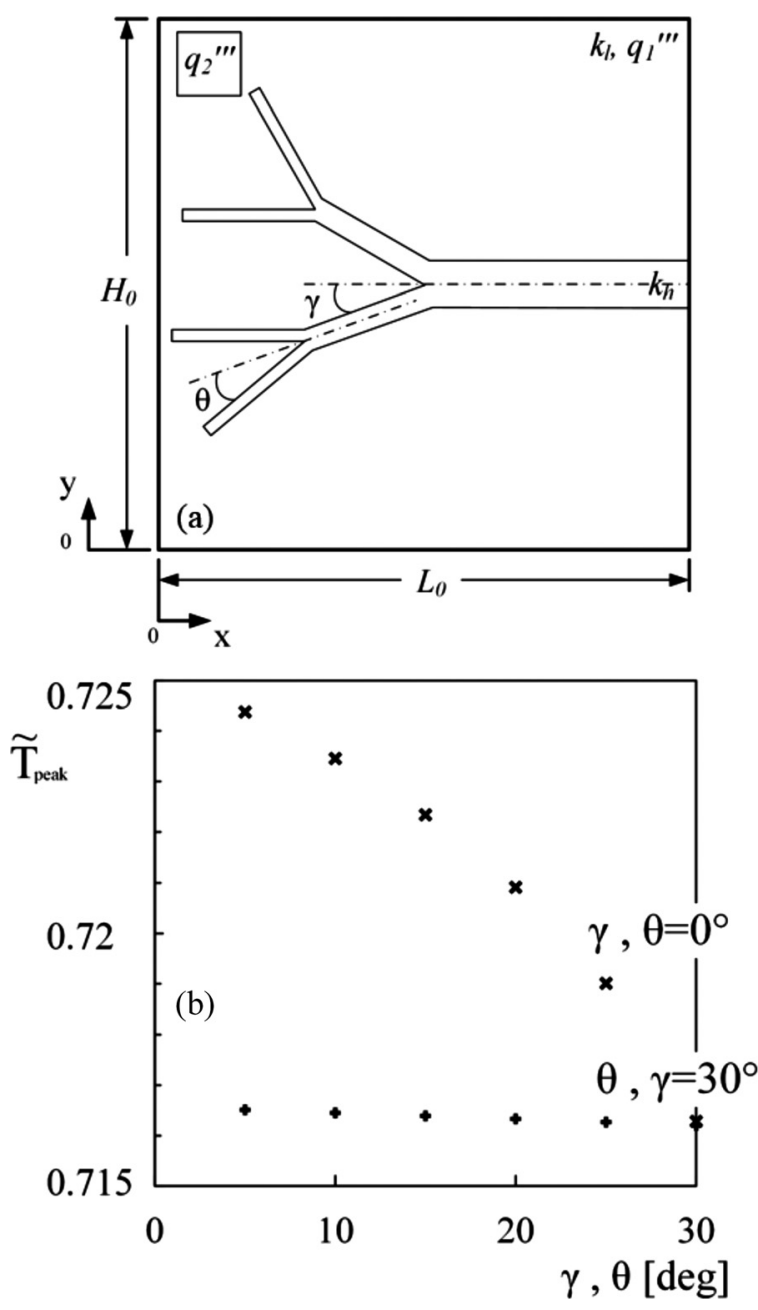

FIG. 7. Heat generating domain with embedded asymmetric Y-shaped pathways: (a) geometry and (b) $\widetilde{T}_{\text {peak }}$ relative to $\theta$ with $\gamma=30^{\circ}$, and $\widetilde{T}_{\text {peak }}$ relative to $\gamma$ with $\theta=0^{\circ}$. 
heat generation shown with heating rates of $q_{1}^{\prime \prime \prime}$ and $q_{2}^{\prime \prime \prime}$. Figure 7 shows how the location of tree-branches located at $y<H_{0} / 2$ affects $\widetilde{T}_{\text {peak }}$ with concentrated hot spot. Figure 7(b) shows that varying $\gamma$ and $\theta$ has almost no effect on $\widetilde{T}_{\text {peak }}$, i.e., around $1.4 \%$ change in $\widetilde{T}_{\text {peak }}$. Therefore, Figure $7(\mathrm{~b})$ suggests that $\widetilde{T}_{\text {peak }}$ is governed by the upper branch, located at $y>H_{0} / 2$. Therefore, the volume fraction of the upper branches should be increased in comparison with the lower branches in order to minimize $\widetilde{T}_{\text {peak }}$ for given boundary conditions. Figure 7(b) also shows that the highconductivity tree should grow in the direction of where heat is generated, and the thickness of the branches located closer to the heat generation should be greater. To sum up, asymmetric trees emerge naturally in order to minimize resistances with non-uniform boundary conditions.

\section{CONCLUSIONS}

This paper shows that the peak temperature of a nonuniformly heated domain can be decreased by only changing the shape of the fixed amount of high-conductivity material. Increasing the number of tree branches decreases the peak temperature. In addition, the high-conductivity inserts should be placed such that the distance between them and the heat generating regions is the smallest. Furthermore, the decrease in the order of peak temperature is smaller as number of tree branches increases. Therefore, increasing the number of tree branches after three levels of bifurcation does not affect the order of peak temperature. This paper also shows that for a given $\phi$ the number of branches corresponding for the minimum peak temperature varies. For instance, decreasing the number of tree branches provides greater overall thermal conductance when $\phi_{0}=0.015$.

This paper also shows that asymmetry emerges naturally with non-uniform boundary conditions. High-conductivity inserts tend to grow toward where the heat is generated in order to minimize the flow resistances. This phenomenon is similar to how tree roots grow toward water and mineral sources in the soil. ${ }^{26,27}$ In summary, we showed that there is a design which provides the smallest peak temperature for a given set of constraints and boundary conditions. There is no optimum design but the best design which provides the smallest peak temperature for given conditions.

\section{ACKNOWLEDGMENTS}

The authors thank Professor Adrian Bejan for his suggestions. Mr. A. Oliani's visit to Izmir Institute of Technology was supported by Erasmus program. Professor E. Cetkin's research was funded from the Scientific and Technological Research Council of Turkey (TUBITAK) under Grant No. 114M592.

\footnotetext{
${ }^{1}$ A. Bejan and S. Lorente, Design with Constructal Theory (Wiley, Hoboken, 2008).

${ }^{2}$ S. Kakac, H. Yuncu, H. Hijikata, and K. Hijikata, Cooling of Electronic Systems (Kluwer Academic Publishers, Dordrecht, The Netherlands, 1994).
}

${ }^{3}$ V. Sajith and C. B. P. Sobhan, "Characterization of heat dissipation from a microprocessor chip using digital interferometry," IEEE Trans. Compon., Packag., Manuf. Technol. 2, 1298-1306 (2012).

${ }^{4}$ E. Cetkin, "Inverted fins for cooling a non-uniformly heated domain," J. Therm. Eng. 1, 1-9 (2015).

${ }^{5}$ M. Rafati, A. A. Hamidi, and M. Shariati Niaser, "Application of nanofluids in computer cooling systems (heat transfer performance of nanofluids)," Appl. Therm. Eng. 45-46, 9-14 (2012).

${ }^{6} \mathrm{M}$. Khoshvaght-Aliabadi and A. Alizadeh, "An experimental study of $\mathrm{Cu}-$ water nanofluid flow inside serpentine tubes with variable straight-section lengths," Exp. Therm. Fluid Sci. 61, 1-11 (2015).

${ }^{7}$ J. M. Jha, S. V. Ravikumar, A. M. Tiara, I. Sarkar, S. K. Pal, and S. Chakraborty, "Ultrafast cooling of a hot moving steel plate by using alumina nanofluid based air atomized spray impingement," Appl. Therm. Eng. 75, 738-747 (2015).

${ }^{8}$ C. S. Sharma, M. K. Tiwari, S. Zimmermann, T. Brunschwiler, G. Schlottig, B. Michel, and D. Poulikakos, "Energy efficient hot spottargeted embedded liquid cooling of electronics," Appl. Energy 138, 414-422 (2015).

${ }^{9}$ R. Jacob, T. Basak, and S. K. Das, "Experimental and numerical study on microwave heating of nanofluids," Int. J. Therm. Sci. 59, 45-57 (2012).

${ }^{10}$ J. B. Marcinichen, D. Wu, S. Paredes, J. R. Thome, and B. Michel, "Dynamic flow control and performance comparison of different concepts of two-phase on-chip cooling cycles," Appl. Energy 114, 179-191 (2014).

${ }^{11}$ M. Almogbel and A. Bejan, "Conduction trees with spacings at the tips," Int. J. Heat Mass Transfer 42, 3739-3756 (1999).

${ }^{12} \mathrm{E}$. Cetkin, "Three-dimensional high conductivity trees for volumetric cooling,” Int. J. Energy Res. 38, 1571-1577 (2014).

${ }^{13}$ G. Lorenzini, C. Biserni, and L. A. O. Rocha, "Constructal design of Xshaped conductive pathways for cooling a heat-generating body," Int. J. Heat Mass Transfer 58, 513-520 (2013).

${ }^{14}$ G. Lorenzini, F. L. Garcia, E. dos Santos, C. Biserni, and L. A. O. Rocha, "Constructal design applied to the optimization of complex geometries: TY-shaped cavities with two additional lateral intrusions cooled by convection,” Int. J. Heat Mass Transfer 55, 1505-1512 (2012).

${ }^{15}$ Z. H. Xie, L. G. Chen, and F. R. Sun, "Geometry optimization of T-shaped cavities according to constructal theory," Math. Comput. Modell. 52(9-10), 1538-1546 (2010).

${ }^{16}$ A. Bejan, Shape and Structure, from Engineering to Nature (Cambridge University Press, Cambridge, UK, 2000).

${ }^{17}$ A. Bejan, Advanced Engineering Thermodynamics, 2nd ed. (Wiley, John \& Sons, Incorporated, 1997).

${ }^{18}$ A. F. Miguel, "Constructal pattern formation in stony corals, bacterial colonies and plant roots under different hydrodynamics conditions," J. Theor. Biol. 242, 954-961 (2006).

${ }^{19}$ A. Bejan, "Science and technology as evolving flow architectures," Int. J. Energy Res. 33, 112-125 (2009).

${ }^{20} \mathrm{~A}$. Bejan and S. Lorente, "Constructal theory and its relevance to green energy,” Int. J. Green Energy 4, 105-117 (2007).

${ }^{21}$ S. Lorente, E. Cetkin, T. Bello-Ochende, J. P. Meyer, and A. Bejan, "The constructal-law physics of why swimmers must spread their fingers and toes," J. Theor. Biol. 308, 141-146 (2012).

${ }^{22}$ J. V. C. Vargas and A. Bejan, "Thermodynamic optimization of internal structure in a fuel cell," Int. J. Energy Res. 28, 319-339 (2004).

${ }^{23}$ A. Bejan, "Thermodynamic optimization alternatives: Minimization of physical size subject to fixed power," Int. J. Energy Res. 23, 1111-1121 (1999).

${ }^{24}$ E. Cetkin, S. Lorente, and A. Bejan, "The steepest S curve of spreading and collecting flows: Discovering the invading tree, not assuming it," J. Appl. Phys. 111, 114903 (2012).

${ }^{25}$ See www.comsol.com for information about COMSOL Multiphysics.

${ }^{26}$ D. Eapen, M. L. Barroso, G. Ponce, M. E. Campos, and G. I. Cassab, "Hydrotropism: Root growth responses to water," Trends Plant Sci. 10, 44-50 (2005).

${ }^{27}$ Y. Bao, P. Aggarwal, N. E. Robbins, C. J. Sturrock, M. C. Thompson, H. Q. Tan, C. Tham, L. Duan, P. L. Rofriguez, T. Vernoux, S. J. Mooney, M. J. Bennett, and J. R. Dinneny, "Plant roots use a patterning mechanism to position lateral root branches toward available water," Proc. Natl. Acad. Sci. 111, 9319-9324 (2014). 